\title{
Tolerance to penicillin in streptococci of viridans group
}

\author{
LISA POWLEY, JULIE MEESON, D GREENWOOD \\ From the Department of Microbiology and PHLS Laboratory, University Hospital, Queen's Medical Centre, \\ Nottingham
}

SUMMARY Tolerance to penicillin was investigated in $\mathbf{4 0}$ isolates of optochin-resistant, $\alpha$-haemolytic streptococci. Thirteen strains exhibited tolerance to penicillin (MBC:MIC ratio $\geq 32$ ) when stationary phase inocula were used, but only seven strains retained the tolerance phenotype in experiments with logarithmic phase inocula. There was a striking association between tolerance and Eagle's optimum dosage effect, particularly among strains that displayed tolerance in both the stationary and the logarithmic growth phases. Sequential viable counts on representative strains showed that reliance on the arbitrary criterion of bactericidal activity of $99.9 \%$ reduction of the original inoculum after 24 hours' exposure may occasionally lead to difficulties in the recognition of penicillin tolerance. In general, however, the $99.9 \%$ killing criterion provided a useful discriminator between strains that were rapidly killed by penicillin and those (tolerant strains) in which the bactericidal activity was much reduced.

Organisms said to be "tolerant" to penicillin or other bactericidal agents are susceptible to inhibition of growth by the antibiotic, but the lethal effect is abolished or much reduced. ${ }^{12}$ The clinical implications of tolerance to penicillin are uncertain, but if the phenomenon has any relevance it is likely to be in those conditions in which the use of bactericidal agents is essential for therapeutic success. One such condition is bacterial endocarditis in which viridans streptococci derived from the normal oral flora are commonly incriminated, and in which benzylpenicillin is the mainstay of treatment. ${ }^{3}$

We examined 40 strains of viridans streptococci isolated from sputum cultures. The isolates were identified to species level and tested for tolerance to benzylpenicillin in broth titrations. Selected isolates were also tested by sequential viable counting. As tolerance may be associated with Eagle's optimal dosage effect ${ }^{45}$ and as the growth phase of the inoculum is known to affect the expression of tolerance $^{6}$ we have examined the association between these factors and tolerance in our isolates.

\section{Material and methods}

\section{BACTERIA}

Forty strains of optochin-resistant, $\alpha$-haemolytic streptococci were randomly isolated from sputum specimens received in Nottingham Public Health Laboratory. The organisms had no clinical relevance other than their potential to cause endocarditis. Each isolate was identified by the API 20 Strep method. Stock cultures were maintained in glycerol blood broth at $-30^{\circ} \mathrm{C}$. In use, cultures were stored at $4^{\circ} \mathrm{C}$ on blood agar plates and subcultured fortnightly.

\section{ANTIBIOTIC TITRATIONS}

Appropriate dilutions of benzylpenicillin (Crystapen, Glaxo Laboratories Ltd) were prepared in ToddHewitt broth. Serial doubling dilutions $(1 \mathrm{ml})$ of the antibiotic were inoculated with $c 10^{5}$ colony forming units (cfu) per $\mathrm{ml}$. The inoculum was derived either from overnight (stationary phase) cultures in ToddHewitt broth, or from logarithmically growing cultures obtained by monitoring the growth of ToddHewitt broth cultures turbidimetrically. Viable counts were performed to establish the actual inoculum in each experiment. After overnight incubation at $37^{\circ} \mathrm{C}$ the minimum inhibitory concentration (MIC) was recorded as the lowest concentration of antibiotic to suppress visible growth. To determine the minimum bactericidal concentration (MBC) one drop of $\beta$-lactamase (ex Bacillus cereus; Koch-Light Ltd) was added to each clear tube with a Pasteur pipette, the tubes were agitated on a vortex mixer, and $0.1 \mathrm{ml}$ was subcultured on to a blood agar plate. The number of survivors (cfu) was counted after overnight incubation at $37^{\circ} \mathrm{C}$. Because the expression of tolerance in streptococci is enhanced in an atmosphere rich in carbon dioxide (Meeson J, Greenwood D, paper presented at Proceedings of the Xth Lancefield International Symposium on streptococci and streptococcal diseases) 
plates were incubated in a candle jar. A bactericidal effect was defined as a $99.9 \%$ reduction in the original inoculum. An MBC:MIC ratio of 32 or more was taken as denoting tolerance.

\section{VIABLE COUNTS}

Cultures of stationary phase or logarithmically growing streptococci were exposed to serial doubling dilutions of benzylpenicillin in Todd-Hewitt broth at $37^{\circ} \mathrm{C}$. At the start of the experiment and at intervals thereafter, samples were removed, vortexed, and counted by the agar droplet method': serial 10-fold dilutions of the cultures were prepared in molten Todd-Hewitt agar at $45^{\circ} \mathrm{C}$ and quintuplicate $0.1 \mathrm{ml}$ drops were delivered into an empty sterile Petri dish. Colonies developing within the agar droplets after incubation for 48 hours at $37^{\circ} \mathrm{C}$ were counted with the help of a magnifier. The number of survivors (cfus) was calculated from droplets containing 10-100 colonies.

\section{Results}

Of the 40 isolates of viridans streptococci, 20 were identified by the API 20 strep system as $S$ sanguis II; the remainder were $S$ sanguis I/1 (six strains), $S$ sanguis I/2 (five strains) and $S$ mitis (nine strains).

The MIC of benzylpenicillin for the 40 isolates ranged from 0.03 to $4 \mathrm{mg} / \mathrm{l}$; the $\mathrm{MBC}$ range was 0.03 to $>64 \mathrm{mg} / \mathrm{l}$ (table 1). For 13 strains, the MIC of benzylpenicillin was $>0.12 \mathrm{mg} / \mathrm{l}$, indicating reduced susceptibility to the antibiotic. There was no correlation between species and MIC, nor between MIC and possession of the tolerance phenotype.

Overall, 13 of the 40 strains (32.5\%) were scored as tolerant (MBC:MIC ratio $\geq 32$ ) when a stationary phase inoculum was used and seven of the 40 strains $(17.5 \%)$ when a logarithmic phase culture was used to provide the inoculum. None of the six $S$ sanguis I/1 isolates was found to be tolerant to penicillin. The proportion of tolerant strains among the other species varied depending on whether a stationary phase or a logarithmic phase culture was used as the source of the inoculum (table 2). All seven strains that exhibited tolerance when tested in the logarithmic growth phase also seemed to be tolerant when stationary phase cultures were used.

Table 1 Distribution of MICs and MBCs of benzylpenicillin among 40 isolates of viridans streptococci

\begin{tabular}{llll}
\hline $\begin{array}{l}\text { Species } \\
\text { (No of isolates) }\end{array}$ & $\begin{array}{l}\text { MIC range } \\
(\mathrm{mg} / \mathrm{l})\end{array}$ & $\begin{array}{l}M B C \text { range } \\
(\mathrm{mg} / \mathrm{l})\end{array}$ & $\begin{array}{l}\text { No of strains with } \\
M B C: M I C \text { ratio } Z 32\end{array}$ \\
\hline S sanguis I/1 (6) & $0.06-4$ & $0.06-8$ & 0 \\
$S$ sanguis I/2(5) & $0.03-2$ & $0.03->64$ & 3 \\
$S$ sanguis II (20) & $0.03-2$ & $0.03->64$ & 6 \\
S mitis (9) & $0.03-2$ & $0.03->64$ & 4 \\
\hline
\end{tabular}

Table 2 Association between penicillin tolerance and growth phase among 40 isolates of viridans streptococci

\begin{tabular}{|c|c|c|}
\hline \multirow[b]{2}{*}{ Species } & \multicolumn{2}{|c|}{ No tolerant when inoculum was in } \\
\hline & $\begin{array}{l}\text { Stationary } \\
\text { phase }\end{array}$ & $\begin{array}{l}\text { Logarithmic } \\
\text { phase }\end{array}$ \\
\hline $\begin{array}{l}S \text { sanguis } \mathrm{I} / 1(\mathrm{n}=6) \\
S \text { sanguis } \mathrm{I} / 2(\mathrm{n}=5) \\
S \text { sanguis } \mathrm{II}(\mathrm{n}=20) \\
S \text { mitis }(\mathrm{n}=9) \\
\text { All species }(\mathrm{n}=40)\end{array}$ & $\begin{array}{r}0 \\
3 \\
6 \\
4 \\
13\end{array}$ & $\begin{array}{l}0 \\
2 \\
5 \\
0 \\
7\end{array}$ \\
\hline
\end{tabular}

The percentage of colony forming units surviving overnight exposure to $64 \mathrm{mg}$ benzylpenicillin/l was 0.02 or less for most non-tolerant strains. The range of survivors among tolerant strains was 0.1 to $0.63 \%$ with a mean of $0.28 \%$. The mean survival of the 13 tolerant strains was reduced to $0 \cdot 15 \%$ in experiments in which logarithmic phase cultures were used as inoculum. Even for the seven strains that remained tolerant under these conditions, the mean survival was reduced from $0.33 \%$ to $0.26 \%$.

\section{EAGLE EFFECT}

With many non-tolerant strains the number of survivors after overnight incubation was too small to assess the presence or absence of any optimal dosage effect. The Eagle effect, however, was not displayed by any of 13 non-tolerant strains in which a sufficient number of survivors was detected.

In experiments with stationary phase inocula seven of the 13 tolerant strains exhibited the Eagle effect in that substantially greater numbers of survivors were detected in high concentrations of penicillin ( $>16$ MIC) than in concentrations of 2 to 4 MIC. Six of these seven isolates also displayed the tolerance phenotype and the Eagle effect when logarithmic phase organisms were used as inoculum. Conversely, only one of six tolerant isolates that did not exhibit the Eagle effect remained tolerant in experiments with logarithmic phase inocula (fig 1).

The mean survival percentages for the seven strains that exhibited the Eagle effect were greater than those of the six negative strains in experiments with both stationary phase and logarithmic phase inocula (table 3).

\section{KILLING CURVES}

Three strains were examined by sequential viable counting, representing a tolerant isolate ( $S$ sanguis II), a non-tolerant isolate ( $S$ sanguis $\mathrm{I} / 1)$, and an isolate that exhibited the tolerance phenotype only when a stationary phase inoculum was used ( $S$ sanguis $\mathrm{I} / 2$ ). The results are shown in fig 2 . When tested with a stationary phase inoculum, the tolerant $S$ sanguis II isolate was clearly tolerant at all concentrations of penicillin tested in that the viable count was reduced 

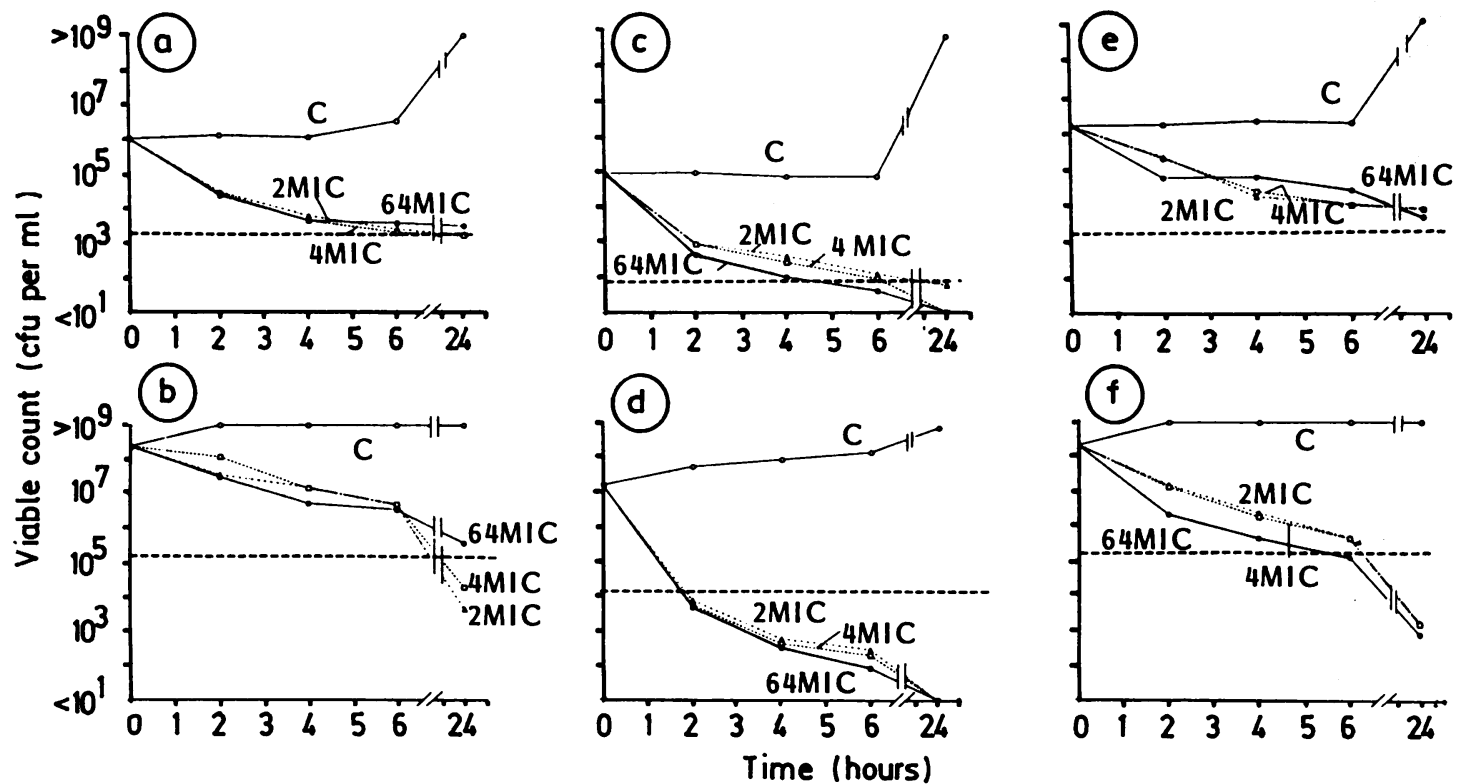

Fig 1 Sequential viable counts of three strains of viridans streptococci exposed in stationary growth phase $(a, c, e)$ or logarithmic growth phase $(b, d, f)$ to benzylpenicillin. $(a, b): S$ sanguis $I I$ (tolerant); $(c, d): S$ sanguis $I / I$ (non-tolerant); (e, $f): S$ sanguis $I / 2$ (tolerant only when tested in the stationary growth phase). Dotted line indicates threshold of tolerance (99.9\% reduction in the original inoculum) according to conventional criteria.

less than 1000-fold during incubation for 24 hours. When a logarithmically growing culture of this organism was tested in the same way, however, tolerance

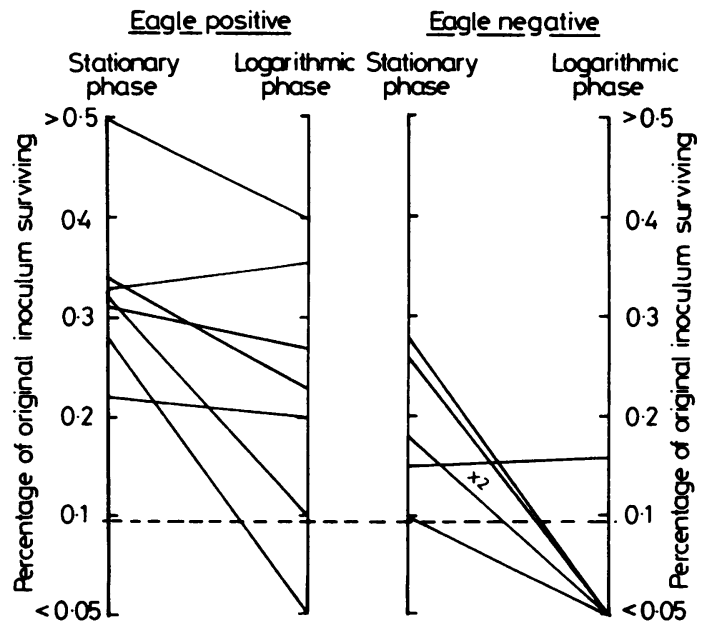

Fig 2 Comparison of percentage of original inoculum surviving exposure to benzylpenicillin for 24 hours when tested in stationary phase and logarithmic phases of growth. Left hand panel: seven strains that exhibited Eagle's optimum dosage effect; right hand panel: six strains that did not show the Eagle effect. Dotted line indiates threshold of tolerance according to conventional criteria. was only evident because of the Eagle effect: concentrations of benzylpenicillin corresponding to 2 or 4 MIC reduced the inoculum about 10000 -fold, but a higher concentration of $64 \mathrm{MIC}$ achieved less than $\mathbf{9 9 . 9 \%}$ killing. At higher concentrations of penicillin the bactericidal effect was further reduced (data not shown).

In contrast, the non-tolerant $S$ sanguis $\mathrm{I} / 1$ was rapidly killed when a logarithmic phase inoculum was used, the viable count being reduced more than 1000 fold within four hours. In experiments with a stationary phase inoculum the rate of killing was reduced but still exceeded the $99.9 \%$ killing threshold for bactericidal activity after overnight exposure. $S$ sanguis $\mathrm{I} / 1$, which exhibited tolerance only when tested in the

Table 3 Association between Eagle effect and survival after exposure to penicillin in 13 tolerant strains of streptococci

\begin{tabular}{lll}
\hline & \multicolumn{2}{l}{$\begin{array}{l}\text { Mean percentage of inoculum surviving in } \\
\text { experiments in which the inoculum was in: }\end{array}$} \\
\cline { 2 - 3 } Eagle effect & Stationary phase & Logarithmic phase \\
\hline Present $(\mathrm{n}=7)$ & 0.35 & $0.23^{*}$ \\
Absent $(\mathrm{n}=6)$ & 0.19 & $0.05 \dagger$
\end{tabular}

-Includes one strain which did not display tolerance or the Eagle effect when tested in the logarithmic phase.

tIncludes one strain which remained tolerant when tested in logarithmic phase. 
stationary phase, was killed more slowly than the nontolerant $S$ sanguis $\mathrm{I} / 1$, even when a logarithmic phase culture was tested.

\section{Discussion}

In an earlier investigation of various types of streptococci ${ }^{5}$ we found that about half of a small number of viridans streptococci tested exhibited tolerance to penicillin. In this study we have confirmed and extended these findings and have shed further light on the tolerance phenomenon in streptococci of the viridans group. Although the numbers examined were too small to allow firm conclusions to be drawn about the prevalence of tolerance among individual species, it was noteworthy that none of six strains belonging to the $S$ sanguis $\mathrm{I} / 1$ subtype exhibited tolerance; nor did any of nine strains of $S$ mitis tested in experiments with logarithmic phase inocula.

The influence of growth phase on the expression of the tolerance phenotype has been reported by others ${ }^{6}$ and is not surprising in view of the fact that penicillin and other inhibitors of bacterial cell wall synthesis require active cell growth in order to achieve their bactericidal effect. ${ }^{8}$ In attempting to relate the findings to the therapeutic situation it should be borne in mind that in vivo active bacterial multiplication probably takes place in established lesions, but not at a logarithmic rate.

The present results also extend further an earlier observation of the relation between tolerance and Eagle's optimum dosage effect. ${ }^{45}$ In the present investigation not only was an association between the two phenomena observed, but there was a significant correlation between the number of survivors and the presence of the Eagle effect (table 3). Moreover, six of seven tolerant strains that exhibited the Eagle phenomenon also displayed the tolerance phenotype in both the logarithmic and stationary phases of growth.

Three representative strains were tested by sequential viable counting. The results illustrate the arbitrary nature of the $99.9 \%$ killing criterion usually applied to bactericidal activity. Thus penicillin caused a considerable fall in bacterial viability in all three strains with both stationary phase and logarithmic phase inocula and in several cases the degree of killing achieved after 24 hours' exposure fell close to the $99.9 \%$ threshold. In experiments with the tolerant $S$ sanguis II strain in which logarithmic phase inocula were used, the decision to score the result as "tolerant" was influenced by the presence of the Eagle effect (fig $2 b)$.

In the case of streptococci and staphylococci the assessment of viability, and hence the expression of tolerance, is complicated by the fact that viable colony forming units may survive within chains or clumps, ${ }^{9}$ so that the extent of killing may be underestimated. In these circumstances the validity of the $99.9 \%$ killing criterion is even more suspect. None the less strains clearly exist in which the bactericidal activity of penicillin is much reduced compared with fully susceptible strains. This property is characterised by a reduction in the rate of killing by the antibiotic rather than by a failure to kill, and the cut off point of $99.9 \%$ killing after 24 hours' exposure is intended to reflect this. The present results suggest that this arbitrary cut off point is, perhaps fortuitously, a useful one: survival after exposure of fully susceptible streptococci generally fell much below the $\mathbf{9 9 . 9 \%}$ level, while survival in tolerant strains was usually above this level. In only a few strains did the $99.9 \%$ criterion of bactericidal activity produce an equivocal result.

\section{References}

1 Sabath LD, Wheeler N, Laverdiere M, Blazevic D, Wilkinson BJ. A new type of penicillin resistance of Staphylococcus aureus. Lancet 1977; i:443-7.

2 Handwerger S, Tomasz A. Antibiotic tolerance among clinical isolates of bacteria. Rev Infect Dis 1985;7:368-86.

3 Wilson WR. Antimicrobial therapy of streptococcal endocarditis. J Antimicrob Chemother 1987;20(suppl A):147-59.

4 Eagle H, Musselman AD. Rate of bactericidal action of penicillin in vitro as a function of its concentration and its paradoxically reduced activity at high concentrations against certain organisms. J Exp Med 1948;88:99-131.

5 Slater GJ, Greenwood D. Detection of penicillin tolerance in streptococci. J Clin Pathol 1983;36:1353-6.

6 Sherris JC. Problems in in vitro determination of antibiotic tolerance in clinical isolates. Antimicrob Agents Chemother 1986;30:633-7.

7 Sharpe AN, Kilsby DC. A rapid, inexpensive bacterial counting technique using agar droplets. J Appl Bacteriol 1971;34:435-40.

8 Hobby GL, Meyer K, Chaffee E. Observations on the mechanism of action of penicillin. Proc Soc Exp Biol Med 1942;50:281-5.

9 Elliott TSJ, Greenwood D, Rodgers FG, O'Grady F. The response of Staphylococcus aureus to benzylpenicillin. Br J Exp Pathol 1979;60:14-23.

Requests for reprints to: Dr L Powley, Department of Microbiology, PHLS Laboratory, University Hospital, Queen's Medical Centre, Nottingham N67 2UH, England. 